Origins of Life: Self-Organization and/or Biological Evolution? (2009) 23-36

(C) EDP Sciences, 2009

DOI: $10.1051 /$ orvie/2009003

\title{
Self-organization phenomena in non-equilibrium chemical systems
}

\author{
J. Boissonade \\ Centre de Recherche Paul Pascal, Université of Bordeaux and CNRS, 115 Av. \\ Schweitzer, 33600 Pessac, France
}

\begin{abstract}
This article is a brief survey of the temporal and spatial self-organisation phenomena that can occur in chemical systems kept far from equilibrium by a permanent feed of fresh reactants such as bistability, oscillations, traveling waves, Turing patterns and front instabilities.
\end{abstract}

\section{INTRODUCTION}

There are two types of self-organisation in chemical systems. Self-assembly operates at the microscopic level and results from atomic or cluster interactions leading to well ordered equilibrium structures characterized by a minimum of free energy, such as crystals, mesophases, lipidic membranes or carbon nanotubes. On the opposite, chemical dissipative structures, popularized by the Brussels school (Nicolis \& Prigogine, 1977), belong to a large class of phenomena that can occur at macroscopic level when a system, kept far from equilibrium, dissipates energy. In chemistry, these non-equilibrium conditions are normally obtained by a permanent feed of fresh reactants. These structures result from the instability of states that conform to the natural symmetry of systems (e.g. stationary for a constant feed) governed by nonlinear evolution laws that can amplify small fluctuations. Then, the evolution can eventually lead to lower symmetry in time (multistability, oscillations), space (stationary concentration patterns), or both (waves, unstationary patterns). Reaction rates are actually nonlinear laws since the most elementary kinetic law, the so called "mass action law", is the product of the reactant concentrations. To get amplification of fluctuations, at least one kinetic pathway must exhibit autocatalysis so that the production of a species increases directly or indirectly its own production rate. For instance, the chlorite-tetrathionate reaction $(\mathrm{CT})$ can be described by a balance equation

$$
7 \mathrm{ClO}_{2}^{-}+2 \mathrm{~S}_{4} \mathrm{O}_{6}^{2-}+6 \mathrm{H}_{2} \mathrm{O} \rightarrow 7 \mathrm{Cl}^{-}+8 \mathrm{SO}_{4}^{2-}+12 \mathrm{H}^{+}
$$

with a reaction rate $v=k\left[\mathrm{ClO}_{2}^{-}\right]\left[\mathrm{S}_{4} \mathrm{O}_{6}^{2-}\right]\left[\mathrm{H}^{+}\right]^{2}$, so that this reaction appears to be autocatalytic with $\mathrm{H}^{+}$.

In rare cases, the input reactants are in sufficient excess for a closed system to remain far from equilibrium for a limited amount of time (the so-called, "pool chemical approximation"), but in general the system has to be permanently fed with fresh chemicals to get structures and, in all cases, to avoid the parameter shift due to the return to equilibrium. We shall first briefly describe some tools used to produce controlled chemical dissipative structures in the laboratory. Although they are rather

Article available at http://www.origins-and-evolution.org or http://dx.doi.org/10.1051/orvie/2009003 


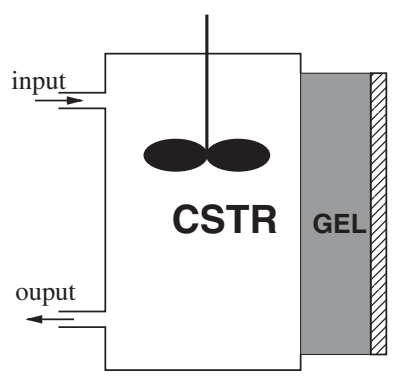

Figure 1. OSFR scheme.

far from the natural systems in which evolutionary processes could have developed, they are convenient to master nonequilibrium parameters and allow for reproducible and quantitative observations.

For temporal instabilities in homogeneous systems, the most popular device is the continuous stirred tank reactor (CSTR), a well stirred tank of constant volume, exchanging the solutions with constant input and output flows characterized by a residence time $\tau$. The state of the system is given by the concentrations $c_{i}$ of species $i$ in the reactor. The control parameters are $\tau$ and the concentrations $c_{0 i}$ in the input flow. The evolution equations are given by

$$
\frac{\mathrm{d} c_{i}}{\mathrm{~d} t}=f_{i}(\mathbf{c})+\frac{1}{\tau}\left(c_{0 i}-c_{i}\right)
$$

where the $f_{i}$ 's are the rate reaction terms and vector $\mathbf{c}$ represents the $c_{i}$ 's.

We shall only consider spatial structures associated to diffusive transport. To avoid convection, the reaction is performed within a chemically inert hydrogel with a high proportion of water (as a 1 or $2 \%$ agarose gel) in order not to significantly alter diffusion properties. The system is fed with fresh reactants by diffusion from gel edges kept in contact with permanently renewed solutions. In most cases, the gel is totally or partially immersed in the contents of a CSTR kept far from equilibrium with a short residence time. A classical set up is the one side fed reactor (OSFR) where only one face of a thin film of gel is in contact with the CSTR contents (Fig. 1). Structures are revealed by an appropriate color indicator. The evolution inside the gel is ruled by reaction-diffusion equations

$$
\frac{\partial c_{i}(t, \mathbf{r})}{\partial t}=f_{i}(\mathbf{c}, \mathbf{r})+D_{i} \nabla^{2} c_{i}(t, \mathbf{r})
$$

where $D_{i}$ is the diffusion coefficient of species $i$. Boundary conditions are given by the concentrations on the gel boundaries at points in contact with feeding solutions and no flux conditions elsewhere.

Modeling relies both on the knowledge or the experimental determination of a kinetic model $\mathrm{d} \mathbf{c} / \mathrm{d} t=f(\mathbf{c})$ for the reaction rates (incuding the flow terms if any) and application of bifurcation theory to search for the instabilities and the generated patterns. The first step is to determine the linear stability of stationary states $\mathbf{c}_{\mathbf{0}}$, i.e. the solutions of $f\left(\mathbf{c}_{\mathbf{0}}\right)=0$. Since $f(\mathbf{c})$ is nonlinear, multiplicity of solutions is possible. One looks at the evolution of a small perturbation $\delta \mathbf{c}=\mathbf{c}-\mathbf{c}_{\mathbf{0}}$ by solving Eq. 1.2 or Eq. 1.3 at first order. In the spatial 
case, the perturbation $\delta c_{\mathbf{k} i}$ for species $i$ can actually be split up into a sum of modes of wavevectors $\mathbf{k}$ in the form $\delta c_{\mathbf{k} i}=\sum_{\mathbf{k}} \exp \left(s_{k i}+j \mathbf{k} \cdot \mathbf{r}\right)$ where the $s_{k i}=\sigma_{k i}+j \omega_{k i}$ are the eigenvalues of the caracteristic matrix, solutions of the system of $i$ equations given by:

$$
\operatorname{det}\left([\mathrm{J}]-\left(s_{k}+k^{2}[\mathrm{D}]\right)=0 \quad \text { with } \quad k=|\mathbf{k}|\right.
$$

Matrices $[\mathrm{J}]=(\partial f / \partial \mathbf{c})_{\mathbf{c}=\mathbf{c}_{\mathbf{0}}}$ and $[\mathrm{D}]$ are respectively the jacobian matrix of kinetic terms at the stationary state and the diffusion matrix which is generally assumed to be diagonal. When there is at least one eigenvalue so that $\sigma_{i k}>0$ the modes of corresponding wavevectors $\mathbf{k}$ are unstable. If $\omega_{i k}>0$ departure from the unstable state is oscillatory. For a system constrained to remain homogeneous, as a CSTR, only the mode $k=0$ has to be considered. If, when one continuously changes a control parameter, there is a change in the number of stationary states or in the stability of some of them, a bifurcation occurs. This results in qualitative changes of dynamical behavior with potential emergence or disparition of temporal or spatial patterns. In generic toy models and close to bifurcations, these behaviors can be studied by elaborated nonlinear techniques such as normal forms, amplitude equations, singular perturbations, etc... For more realistic systems and to simulate real experiments, one has generally to resort to numerical techniques.

It is beyond the scope of this short elementary presentation to enter into technical details. We shall now limit ourselves to a qualitative description of basic phenomena, focusing on the underlying physics. The interested reader is invited to switch to more extensive literature. In this purpose, selected general references will be given in section 8 .

\section{BISTABILITY}

Bistable systems can exhibit two stable stationary states for a same set of control parameters. Many autocatalytic reactions carried on in a CSTR exhibit bistability as a function of the residence time $\tau$ (Pojman \& Epstein, 1998). Let us assume that $X$ is the autocatalytic species produced in the reaction. At short $\tau$, the extent of reaction $\xi$ is low. The reaction time is longer than the time to refill the reactor with fresh reactants and the composition remains close to the concentrations in the input flow (low [X]). On the opposite, for a long $\tau, \xi$ is large and the reaction is almost completed. At intermediate residence times, the situation is more complex since the reaction rate strongly depends on the amount of autocatalytic species already present in the tank. Thus, the final conversion rate, which results from competition with the flow losses, depends on the initial composition in the tank, which is different if one increases $\tau$ from low values or decreases $\tau$ from high values. In a finite domain of $\tau$, both states (low and high $\xi$ ) can be stable and are selected according to the previous history of the system. The two branches, a "flow branch" $F$ with low $\xi$ and a "thermodynamic branch" $T$ with high $\xi$ are sketched in Fig. $2 \mathrm{a}$ as a function of $1 / \tau$. For mathematical reasons, the two branches are connected by an unstable branch. The two points where a stable branch vanishes by collision with an unstable branch are saddle-node bifurcations. By crossing continuouly the bistability domain back and forth one describes an hysteresis cycle. Obviously, another control parameter different from $\tau$ can be chosen to follow these branches. An experimental exemple of bistability in CSTR in the Chlorite-Iodide reaction is presented in Fig. 2b where $\left[\mathrm{I}^{-}\right]_{0}$ was the control parameter and where the electrode potential was used to characterize the state of the system. 

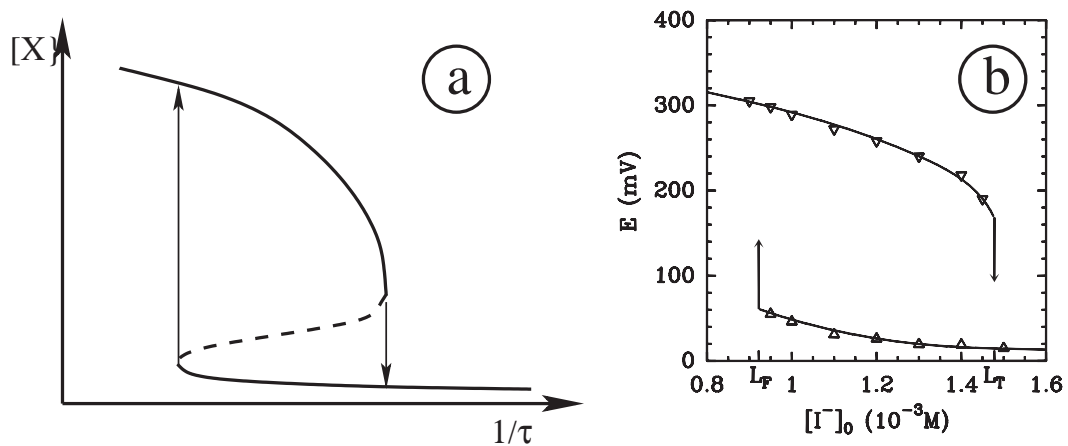

Figure 2. a) Bistability scheme: stable branches (full lines) and unstable branch (dotted line). b) Experimental bistability domain in the $\left(\mathrm{ClO}_{2}, \mathrm{I}^{-}\right)$reaction.

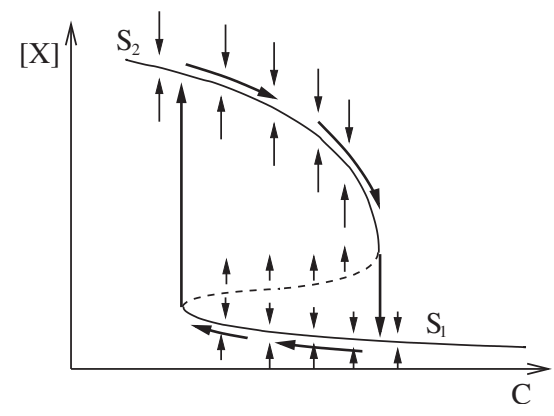

Figure 3. From bistability to oscillatory behavior. $\mathrm{C}$ is a parameter control affected by the feedback.

\section{OSCILLATORY REACTIONS}

Athough sustained oscillations have been known in nonlinear physics for a long time, their existence was ignored and even often denied by chemists until the numerous studies on the Belouzov-Zhabotinskii reaction, referred further on as B.Z., starting in 1963 (Zhabotinsky, 1991). One can understand the existence of oscillations from a physical point of view. Consider a bistable reaction which presents two stable branches $S_{1}$ and $S_{2}$ as explained in the previous section. A representative point of the system moves until it sets on one of the stable branch, say $S_{1}$. If one adds different reactants, the new reactive pathway that is open in the mechanism can create a slow feedback on the control parameter so that the steady state slowly shifts along the branch $S_{1}$ to reach a new stationary state solution. It can find a new stable position on $\mathrm{S}_{1}$, but it can also reach the bifurcation point and jump to the other branch $S_{2}$. Now, the effect of the feedback induces a shift along $S_{2}$. If the feedback is appropriate, it can happen that the system also reaches the bifurcation point on $S_{2}$ and switches back to $S_{1}$ and the process repeats. Since there is no stable stationary state on any branch, the system switches periodically between the two branches and exhibits relaxation oscillations as sketched in Fig. 3. When the feedback time is of the same order than the relaxation time to the $\mathrm{S}$ branches, the oscillations are 
less relaxational, more harmonic and eventually vanish. From a mathematical point of view, oscillatory behavior is associated to a Hopf bifurcation of a stationary state. If the core of the mechanism can be reduced to a two variable model according to

$$
\begin{aligned}
& \frac{\partial u}{\partial t}=f(u, v) \\
& \frac{\partial v}{\partial t}=g(u, v)
\end{aligned}
$$

the Jacobian matrix at the stationary state $\left(\begin{array}{cc}f_{u u} & f_{u v} \\ g_{v u} & g_{v v}\end{array}\right)$ must have at least two positive terms, one on the diagonal (autocatalysis), one outside the diagonal (the positive feedback) and take one of the two following forms: $\left(\begin{array}{c}+_{-}^{-} \\ +-\end{array}\right)$or $\left(\begin{array}{c}++ \\ --\end{array}\right)$ where we assumed the autocatalyic species has concentration $u$. The former corresponds to an activator/inhibitor process where $u$ and $v$ oscillate opposed in phase, the latter to a substrate inhibition where the two species oscillate in phase. When more variable are involved, more complex oscillatory phenomena can occur such as pseudo periodic or chaotic oscillations.

The relations between bistability and oscillations have been formalized into a systematic process to design chemical oscillators (Boissonade \& De Kepper, 1980, Pojman \& Epstein, 1998). A number of oscillating reactions have been produced on these bases. More than a hundred reactions, distributed in about thirty families are known. These are mainly oxydo-reduction reactions involving halogene or sulfur components (Pojman \& Epstein, 1998). Various periodic or complex oscillations in the BriggsRauscher reaction (Briggs \& Rauscher, 1973) are shown in Fig. 4 (from De Kepper, 1980). Only a few biochemical oscillating reactions have been observed in the laboratory. The best studied biochemical oscillator is the glycolysis which can perform oscillations in the CSTR, a well controlled system (Nielsen et al., 1998). These glycolytic oscillations are shown in Fig. 5.

\section{EXCITABILITY AND WAVES}

There is another phenomenon, namely, excitability, which is governed by a mechanism close to the relaxation oscillations described in the former section. It occurs when branch $\mathrm{S}_{2}$ is still unstable under the feedback action but there is a stable stationary state $\mathrm{P}$ located close to the bifurcation point on branch $\mathrm{S}_{1}$ (Fig. 6a).

A small perturbation to state $\mathrm{P}$ is sufficient for the system to leave the attracting domain of $S_{1}$ and to jump to $S_{2}$. Then the slow drift along $S_{2}$ first moves the system to the bifurcation point where it switches back to $S_{1}$. The drift on $S_{1}$ eventually brings back the system to the stable state P. In chemical systems, where there are often very different concentrations and time scales, the jump produces a large pulse that can reach several orders of magnitude (Fig. 6b). The system is no longer excitable until it comes back into the vicinity of $P$.

When diffusive transport is allowed, this leads to spatiotemporal patterns. As soon as a large local pulse has been excited, diffusion quickly brings the pulse vicinity to the threshold and excites the medium around. Thus, the pulse propagates much faster than 

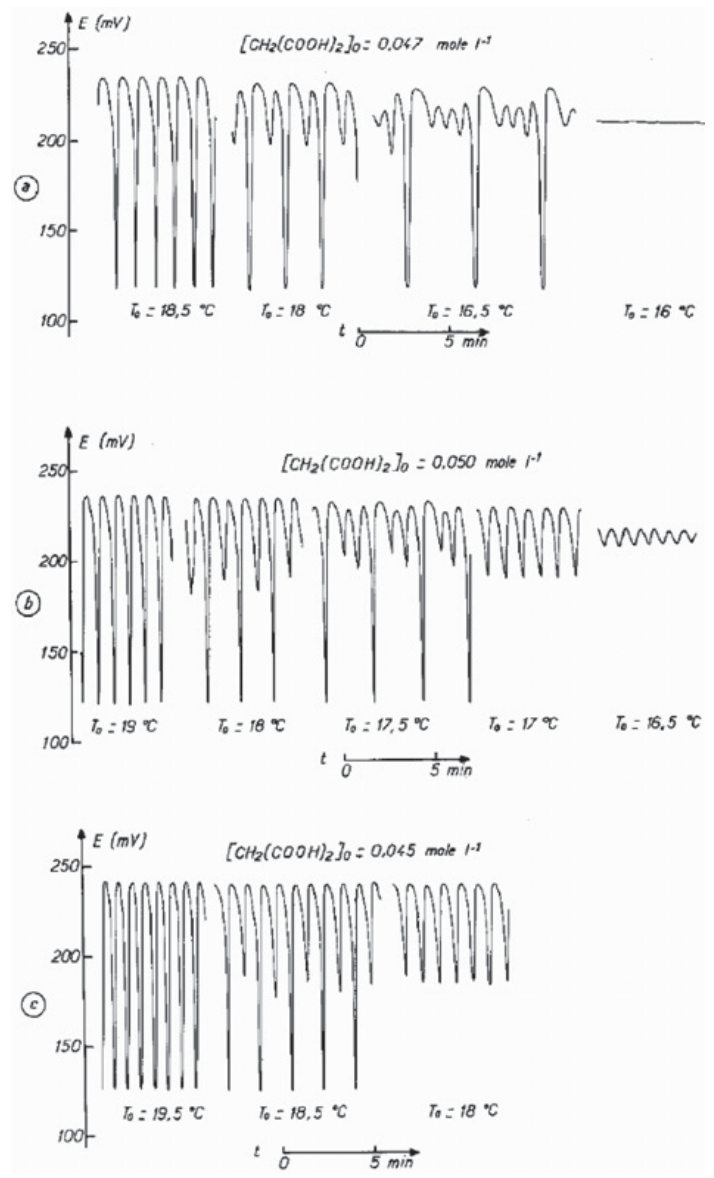

Figure 4. Different forms of oscillations (platinum electrode potential) in the $\left[\mathrm{IO}_{3}^{-}, \mathrm{H}_{2} \mathrm{O}_{2}, \mathrm{Mn}^{2+}\right.$, $\mathrm{CLO}_{4}^{-}$, Malonic acid] reaction (Briggs-Rauscher reaction). From (De Kepper, 1980).

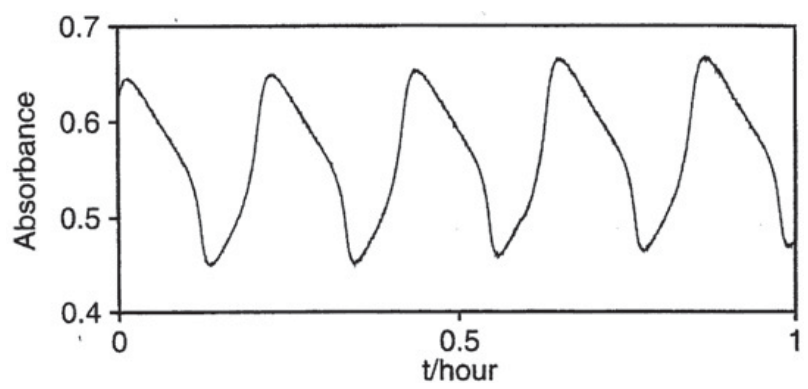

Figure 5. Glycolytic oscillations in a CSTR. From (Nielsen et al., 1998). 

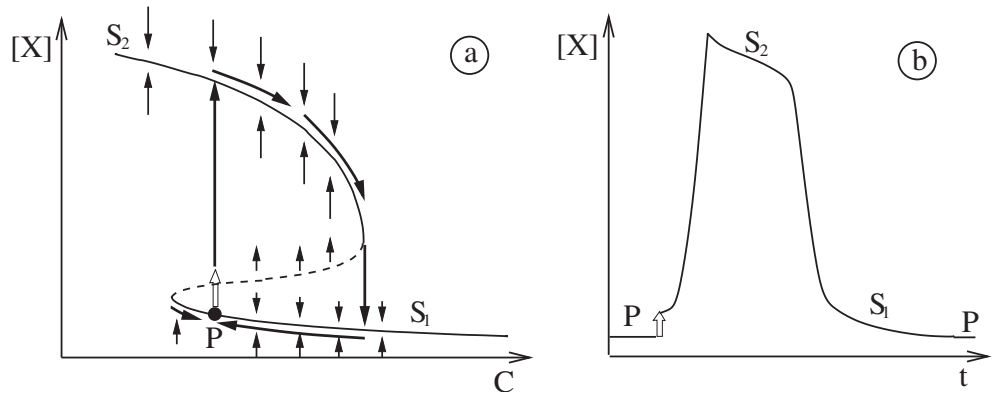

Figure 6. Excitability scheme. Temporal response to a perturbation (white arrow) to the stationary state P.

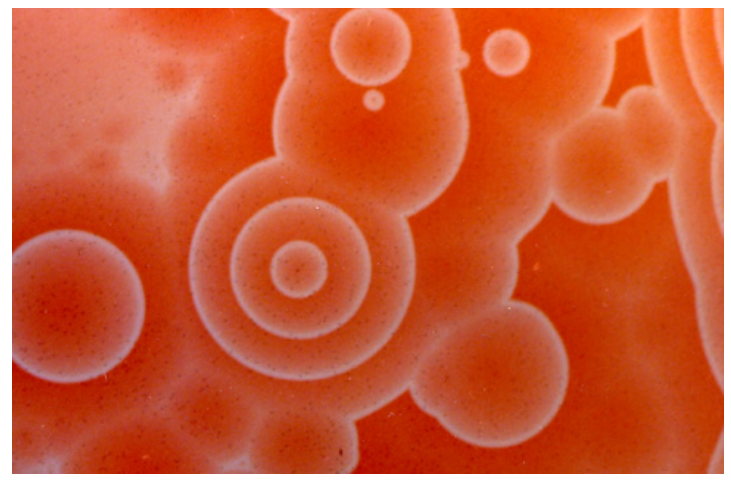

Figure 7. Target patterns in the Belouzov-Zhabotinskii reaction. The typical wavelength is of order $1 \mathrm{~mm}$.

one would expect from simple diffusion and forms traveling waves with a forefront and a backfront. Behind the wave the medium is in a non excitable refractory state until a vicinity of point $\mathrm{P}$ is recovered. When waves meet they annihilate each other.

The most spectacular traveling waves and associated patterns are those of the B.Z. reaction. Because this reaction can approximate pool chemical conditions for a significant amount of time, they can be observed in a closed system (Petri dish) Among the numerous dust particles and impurities that are unavoidably present in the medium, some of them are able to spontaneouly create a perturbation above the threshold and excite a circular wave. As soon as the vicinity of this pacemaker has recovered excitability, a new concentric wave is emitted. The process repeats periodically, with a frequency depending on the pacemaker, giving rise to target patterns (Zaikin \& Zhabotinskii, 1970) shown in Fig. 7. One can show that the velocity $c$ of the wave depends on its local curvature according to the eikonal equation $c=c_{0}-D / R$, where $R$ is the curvature radius and $D$ is a diffusion coefficient. Since the forefront and the backfront move at the same velocity, when one breaks a traveling wave, there must be a stagnation point $\mathrm{P}$ of zero velocity as shown in Fig. 8a. Thus, the wave must coil up into a spiral around this point as shown in Fig. 8b. Breaking a wave gives birth to a pair of counterrotating spirals (Winfree, 1972) as shown 

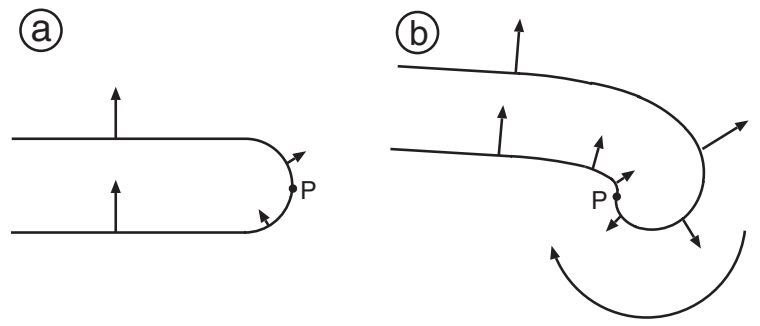

Figure 8. Front motions and spiral formation at a broken wave.

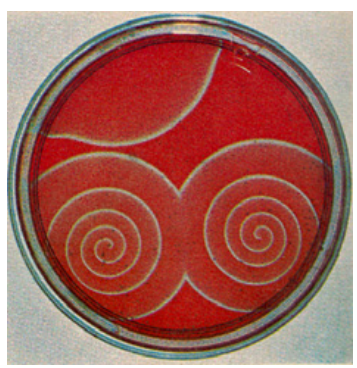

Figure 9. A counterrotating pair of spirals in the B.Z. reaction. From (Winfree, 1974).

in Fig. 9. Such spirals form spontaneously in target patterns when the waves encounter an obstacle such as a gaz bubble produced by the reaction. It is possible to continually sustain a unique spiral when the reaction is operated in an open reactor (OSFR).

\section{TURING PATTERNS}

The existence of Turing patterns has long been a subject of controversies since their prediction by the mathematician A. Turing (Turing, 1952) and was questioned until their first experimental evidence by the Bordeaux group (Castets et al., 1990). These are well organized stationary concentration patterns which result from the sole competition between reaction and diffusion. They rest on an apparent paradox: Although the diffusion is expected to damp fluctuations and to stabilize the medium, it plays the opposite role. This can be understood on the basis of a simple activator/inhibitor model. The activator is an autocatalytic species which increases its own production but also produces its inhibitor (if not, the system would consume all the substrate in an explosive way). If all diffusion coefficients are close to each other, any fluctuation of the activator is balanced by the inhibitor production and is actually damped by diffusion. If the diffusion of the inhibitor is much faster than the diffusion of the activator $\left(D_{h} \gg D_{a}\right)$, the situation is different as illustrated in Fig. 10. When a positive fluctuation of the activator concentration forms in a small zone of a uniform reactive medium (a), the produced inhibitor diffuses outside the zone, limiting the inhibitor increase and leaving the activator to growth (b). This even eventually creates depletion of the activator around this zone (c). An activator peak develops, creating a spatial instability. Then the wings of the various peaks that 
a

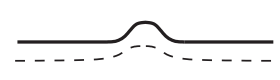

b

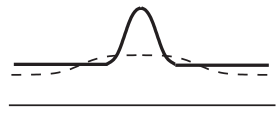

c

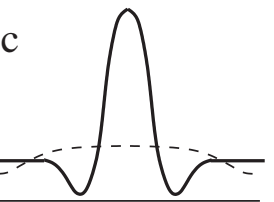

Figure 10. Mechanism of fluctuation growth in the Turing instability. Thick continuous line: activator; dotted line: inhibitor.

come out in the reacting medium begin to interact, leading to well organized structures. Emergence of Turing patterns can be predicted from Eq. 1.4 when the real part $\sigma_{k}$ of one eigenvalue become positive for a nonzero wavevector of modulus $k=k_{c}$. Structures with wavelength $\lambda=2 \pi / k_{c}$ can grow from this instability. It results from the nonlinear interactions that only a few periodic patterns can actually be stable. For instance, it can be shown that, in a two-dimensional system (2-D), only striped (parallel bands) and hexagonal patterns are stable. An important property of Turing patterns is the intrinsic character of the wavelength. Contrary to most nonlinear patterns in physics, $\lambda$ does not depend on the size of the system, but only of the chemical and transport parameters, namely, the concentrations of input species, the kinetic rate constants, and the diffusion coefficients. This allows the system to self-organize with a length scale defined from the sole chemical properties.

It can be seen from Eq. 1.4 that if all diffusion coefficients are equal, the diffusion terms are reduced to a scalar $-D k^{2}$ and contribute negatively to the eigenvalues so that, if any, an instability primarily occurs at $k=0$ and no Turing patterns can develop. The condition for their existence are in agreement with our heuristic presentation: There must be an activatory and an inhibitory pathway - i.e. the same conditions that allow for oscillatory behavior - and at least one of the species that controls the activation must diffuse slower than the species that controls the inhibition. This stringent condition has been the main hindrance in the experimental search. Actually, all small molecules have similar diffusion coefficients $D \sim 10^{-5} \mathrm{~cm}^{2} / \mathrm{s}$. To overcome this problem, one had to introduce in the gel a macromolecule that is too big to diffuse within it and is able to create a nonreactive complex SX with the autocatalytic species X through the equilibrium

$$
\mathrm{S}+\mathrm{X} \leftrightarrows \mathrm{SX}
$$

Activator molecules are immobilized when they are locked in the complex so that their effective diffusion appears to be much smaller than the normal diffusion. One can even make $\mathrm{H}^{+}$, the fastest diffusive ion, to diffuse slowler than the other species. This also modifies the effective kinetics but does not preclude the existence of the patterns.

The [Chlorite-Iodide-Malonic Acid] reaction (referred as CIMA) or its variant the [Chlorine dioxide-Iodine-Malonic Acid] reaction (referred to as DCIMA) exhibit Turing patterns. The complexing agent is the color indicator (starch or polyvinylalcool). In Fig. 11 are shown the CIMA reaction patterns produced in a biface gel, i.e. the reactants are divided in two non reactive solutions that are kept in contact with the two opposite faces of a disk of gel. The structures arise in a film layer parallel to the faces where the concentrations meet the instability conditions. In Fig. 11(a,b,d) the layer is of the same order as the wavelength (typically $0.2 \mathrm{~mm}$ ) so that the system is quasi 2-D and the standard 

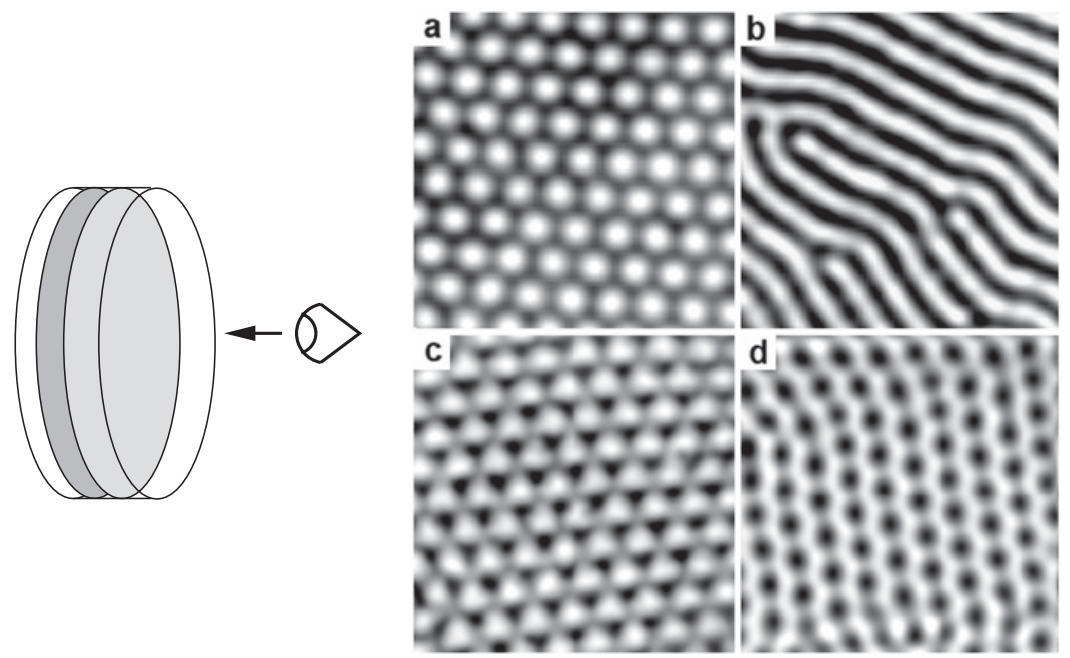

Figure 11. Turing patterns of the CIMA reaction in a biface gel disk (Scheme and observation direction shown on left). $a, d$ : 2-D hexagonal patterns; $b$ : 2-D striped pattern; $c$ : projection of a pattern of larger dimensionality (see text).

striped and hexagonal patterns are observed. In Fig. 11c, the layer is slightly thicker so that an apparently exotic pattern result from the integration of the light absorbance through the gel thickness.

In the CDIMA reaction, the concentrations of iodide $\left[\mathrm{I}_{2}\right]$, chlorine dioxide $\left[\mathrm{ClO}_{2}\right]$, malonic acid [MA] and complexing agent [S] can be considered as constant on a wavelength scale and on the typical time scale. On this basis, Lengyel and Epstein (1991) have shown that the dynamical equations for the reaction could be approximated by a simple two variable model

$$
\begin{gathered}
\frac{\partial u}{\partial t^{\prime}}=\frac{1}{\sigma}\left(a-u-4 \frac{u v}{1+u^{2}}+\Delta_{\mathbf{r}^{\prime}} u\right) \\
\frac{\partial v}{\partial t^{\prime}}=b\left(u-\frac{u v}{1+u^{2}}\right)+d \Delta_{\mathbf{r}^{\prime}} v
\end{gathered}
$$

where $u \propto\left[\mathrm{I}^{-}\right], v \propto\left[\mathrm{ClO}_{2}^{-}\right], t^{\prime}$ and $\mathbf{r}^{\prime}$ are renormalized time and space coordinates respectively. The proportionality factors and all other quantities only depend on the kinetic contants and the constant concentrations above. The activator role is played by $\mathrm{I}^{-}$(variable $u$ ) - actually by substrate inhibition - through the rational fraction terms. At large $u$ the rate at which $\mathrm{I}^{-}$is consumed behave like $1 / u$ and decreases when $u$ increases. The effective diffusion of $\mathrm{I}^{-}$is controlled by $\sigma$, a term that increases linearly with the complexing agent concentration [S]. This simple model allows for analytic linear and nonlinear predictions. Less simplified versions based on balance equations have been used for numerical simulation of quasi-two dimensional patterns in parallel with experimental studies performed in an OSFR analog to the one of Fig. 1 (Rudovics et al., 1999). 


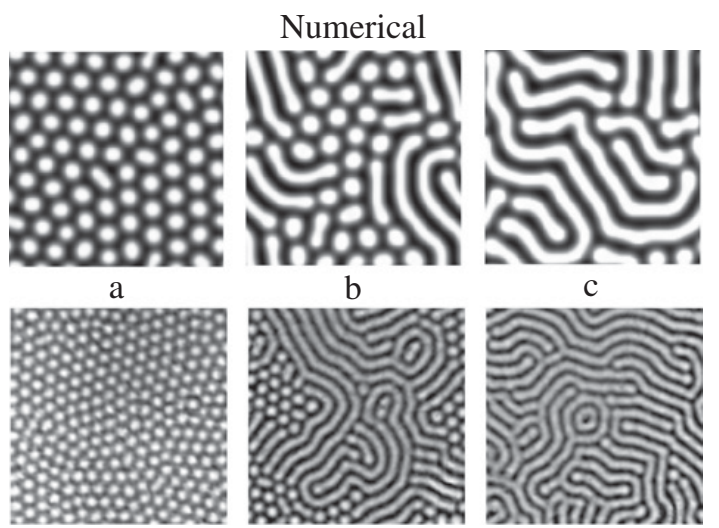

Experimental

Figure 12. Experimental and numerical sequences of Turing patterns of the CDIMA reaction in an OSFR when the MA concentration is increased. From (Rudovics et al., 1999).

In Fig. 12 is shown a sequence of experimental patterns and the corresponding numerical sequence in this OSFR. When striped and hexagonal patterns are both stable, domains of each type coexist in the gel (Fig. 12b). Up to now, only the CIMA and its CDIMA variant had produced experimental Turing patterns. Recently, a second one, namely, the [Thiourea, Iodate, Sulfite] reaction, has also been shown to exhibit such patterns (Horvath et al., 2009) on the basis of a systematic approach.

\section{OTHER PATTERNS}

There are a number of other temporal and spatial chemical patterns. References and developments will be found in the various reviews mentioned in the last section. All these patterns share the property of having at least an autocatalytic pathway. Some of them are extensions of the phenomena discussed above, like chaotic oscillations. Bistability can be generalized to spatial behavior, i.e. several spatial distribution of concentrations can be stable for the same boundary conditions. Whereas long range inhibition is the source of Turing patterns, long range activation, where the activator diffuses faster than the inhibitor, can induce oscillatory phenomena in a non-oscillatory medium. Propagation of reaction fronts can lead to original dynamical behaviors when autocatalytic processes are associated to significant differences in diffusion coefficients. In these conditions, the front can develop transverse instabilities and exhibit simple or complex geometrical modulations. Whereas traveling fronts of Section 4 annihilate themselves in collisions, differential diffusion can also confer to plane or curved fronts a repulsive and self avoiding character which, in turn, may induce the formation of labyrinthine patterns. Differential diffusion is also at the origin of spatiotemporal patterns made of self replicating spots which permanently divide or vanish. A variety of structures which have been observed in OSFR reactors are gathered in Fig. 13. 


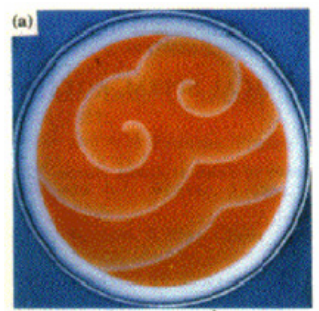

Spiral

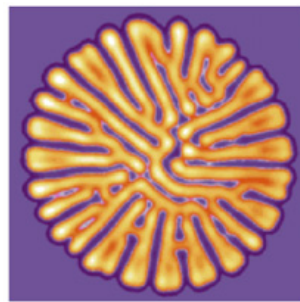

Chemical "flower"

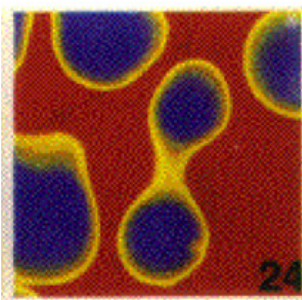

Replicating spots

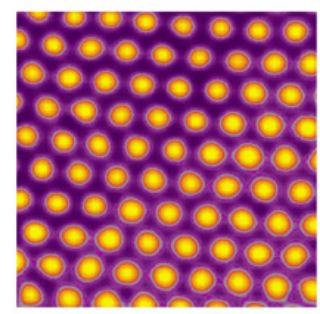

Turing (hexagons)

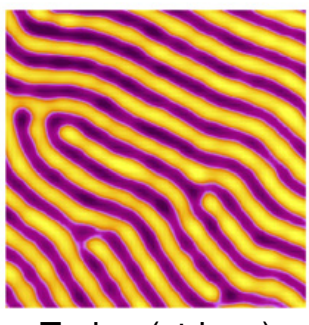

Turing (stripes)

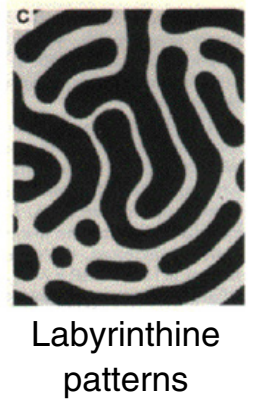

Figure 13. Various patterns observed in an OSFR.

\section{GENERAL OR EXOTIC PHENOMENA?}

The relevance of chemical dissipative system to biological evolution is questionable. However, their apparently exotic character is deceptive.

One could think that the conditions for the appropriate instabilities to occur are extremely stringent. For instance, one knows only two reactions able to produce Turing structures. However, these restrictions are mainly imposed by the experimental facilities: One needs to control the nonequilibrium constraints and to collect the data in well reproducible conditions at convenient time and size scale with easily available (and cheap!) reactants. For all these reasons, one essentially uses small inorganic molecules diffusing easily in hydrogels. Unfortunately, there are few autocatalytic processes in reactions that involve these molecules in solution, whereas they are common in biology where all processes are out of equilibrium since they need a permanent supply of chemical energy. Many metabolic control steps, such as most enzymatic reactions, are governed by 
activation/inhibition processes. While small molecules have similar diffusion coefficients, biological molecules have a large spectrum of sizes, ranging from small fastly diffusing ions to huge proteins. Moreover, the transport in membranes or cells is not purely Fickian and involves complex transfer mechanism that create large differences in the effective diffusion rates. Thus, although they are difficult to be reproduced and controlled in the laboratory, favourable conditions for emergence of patterns in biological systems seem to be the rule rather than the exception.

Moreover, we have focused on diffusive transport since it is the simplest and prototypal mechanism to couple the reaction with spatial properties but there are many other physical processes that can be at the origin of instability and patterns when coupled with reaction, like hydrodynamical or mechanical interactions. For instance, it has recently been shown that a bistable reaction can become oscillatory and induce mechanical oscillations if it is coupled with the swelling properties of a gel immersed in a stationary medium (Borckmans et al., 2009). Since chemical dynamics are already nonlinear, even a linear physical property - this the case of diffusion - can be used. Moreover, in spite of an apparent diversity, the bifurcations and the related instabilities are universal and common to all nonlinear systems. For instance, in systems which can be reduced to two variables, there are only two generic bifurcations - i.e. robust in regard to small changes in the system - namely, the saddle-node (implied in bistability) and the Hopf bifurcation (source of oscillatory behavior). The dynamical behaviors have also universal character. Spiral waves can be found in domains as much different as the B.Z. reaction, cardiac waves, agregation in populations of slime molds, or catalytic oxydation on platinum surfaces. Whereas detailed modeling generally implies a numerical approach, many qualitative predictions can be done on the basis of these universal behaviors.

Chemical dissipative structures should more likely be involved in the formation of biological patterns at the level of the cell, in tissues organisation or in population dynamics rather than in the very early stages of evolution. Nevertheless, they could play an important role in the formation of templates for the expression of the genetic code or adaptation to the surrounding medium. There are a growing number of models relating oscillating reactions or traveling waves with biological rhythms and forms (Goldbeter, 1996, Murray, 2007). It would be surprising that they would not have played some role in the evolution. However, this would be more at the macroscopic level (starting with a few microns) and in the selection process than at the genetic code level.

\section{TO LEARN MORE}

In this introductory paper, the bibliography has been restricted to a few general references. The interested reader will find more general development and a more complete bibliography in the following books and review articles.

Pojman \& Epstein (1998) have written a general textbook for a large scientific audience. It covers all aspects of chemical dissipative structures. The two volumes by Murray (2007) are more technical and develop both mathematical aspects and a great number of models applicable to biological systems. Goldbeter (1996) also considers applications of oscillating reactions to biology. The feature articles gathered in (Field \& Burger, 1985) and (Showalter \& Kapral, 1995) have been important milestones in the literature of the field. Other recent reviews can be found in the proceedings of a summer 
school (Borckmans et al., 2009). The textbook of Mikhailov (1994) covers nonlinear topics which are not limited to chemistry but contains an excellent chapter on traveling waves. De Wit (1999) has written a good review on Turing structures. Various phenomena touched on in Section 6 are described in a feature article by Boissonade et al. (2006). The general review article of Saguès \& Epstein (2004) is slightly more developed than the present one and contains an extensive bibliography.

Some illustrations of this article emanate from previous experimental works achieved at the Centre de recherche Paul Pascal by P. De Kepper and E. Dulos to whom I am specially indebted for their communication.

\section{References}

Boissonade, J., De Kepper, P. 1980, J. Phys. Chem, 84, 501.

Boissonade, J., De Kepper, P., Gauffre F., Szalai I., 2006, Chaos, 16, 037110.

Borckmans, P., De Keppper, P., Kholkhov, A., Métens, S. 2009, editors, Chemomechanical Instabilities in Responsive Material (Springer), in press.

Briggs, T.S., Rauscher, W.C. 1973, J. Chem. Ed., 50, 496.

Rudovics, B., Barillot, E., Davies, P.W., Dulos, E., Boissonade, J., De Kepper, P. 1999, J. Phys. Chem., A103, 1790.

Castets, V., Dulos, E., Boissonade, J., De Kepper, P., Phys. Rev. Lett., 64, 2953.

De Kepper, P. 1980, PhD Thesis, Université Bordeaux.

De Wit, A. 1999, Adv. Chem. Phys., 109, 435.

Field R.J., Burger, M., editors 1985, Oscillations and Traveling Waves in Chemical Systems (Wiley, New York).

Goldbeter A. 1996, Biochemical Oscillations and Cellular Rhythms: The Molecular Bases of Periodic and Chaotic Behaviour (Cambridge Univ. Press).

Horvath, J., Szalai, I., De Kepper, P. 2009, Science, 324, 772.

Nielsen, K., Sorensen, P.G., Hynne, F., Busse, H.-G. 1998, Biophys. Chem., 72, 49.

Lengyel, I., Epstein, I.R. 1991, Science 251, 650.

Mikhailov, A. 1994, Foundations of Synergetics I (Springer, Berlin).

Murray, J. 2003, Mathematical Biology I \& II (Springer, Berlin, New-York).

Nicolis, G., Prigogine, I. 1977, Self-organization in non-equilibrium systems (Wiley, New-York).

Pojman, J.A., Eptein, I.R. 1998, An Introduction to Nonlinear Chemical Dynamics (Oxford University Press, New York, Oxford).

Saguès, F., Epstein, I.R. 2003, Dalton Trans., 1201.

Showalter, K., Kapral, R., editors 1995, Chemical Waves and Patterns (Kluwer, Dordrecht, Boston, London).

Turing, A. 1952, Philos. Trans. R. S. London B, 327, 37.

Winfree, A.T., 1972, Science, 175, 634.

Winfree, A.T. 1974, Scientific American, 230 (6), 82.

Zhabotinskii, A. 1991, Chaos, 1, 379.

Zhaikin, A.N., Zhabotinskii, A. 1970, Nature, 225, 535. 